\title{
SHELLS AND ENCOUNTERS OF DISK GALAXIES WITH ELLIPTICALS
}

\author{
Tsvi Piran \\ Racah Institute for Physics, Hebrew University, Jerusalem, Israel \\ \& The Institute for Advanced Study, Princeton, NJ 08540, USA \\ Jens Verner Villumsen \\ California Institute of Technology, Pasadena, CA 91125, USA
}

\section{INTRODUCTION}

Faint shells have been found around a large number of ellipticals (Malin and Carter 1980, 1983; Schweizer, 1980). Quinn (1984) has suggested that shells form around ellipticals when a small disk galaxy is tidally disrupted in a near radial orbit. According to this mechanism the remnants of the nearly two-dimensional disk maintain their dimensionality and form sheets. These sheets will phasewrap around the elliptical, and the shells are seen when the line of sight is tangent to one of these surfaces (Quinn 1984, Hernquist and Quinn 1986a,b,c).

A shortcoming of Quinn's analysis is that the disruption of the disk galaxy is not treated selfconsistently. The disruption of the disk galaxy is done "by hand". The stars of the disk galaxy are treated as test particles in the fixed potential of the elliptical galaxy. After the disk galaxy has been disrupted, self gravity of the disk is unimportant, but it is important during the disruption phase.

Another problem is that the disks have zero thickness. This helps the contrast seen in the shells. Some estimates have been made of the effects of finite thickness, and the conclusion is that the finite thickness is not a problem (Quinn 1984).

\section{NUMERICAL METHODS}

We have used a new numerical method, based on expansion of the potential in bispherical coordinates. Solution of the Laplace equation using a Fourier transform is one of the common methods for $\mathrm{N}$-body simulations. This fast method is best suited for a general code, where there is no particular geometry. When the configuration studied has a particular geometry it might be favorable to employ suitable coordinates and solve the Laplace equation using an expansion with the appropriate special functions (Villumsen 1982). Overall, such computations can be much faster than those done with a general purpose code. For the study of interaction between two galaxies the bispherical coordinates (e.g. Morse and Feshbach, 1953) seems the best. In our code the focal points of the bispherical coordinates move along in the computation and follow the centers of the galaxies. We use these coordinates to calculate the gravitational forces. The integration of the particles' orbits is carried out in Cartesian coordinates with a fourth order Adams-Moulton predictor corrector. 


\section{DISCUSSION}

We test Quinn's model with fully self consistent 3-D N-body simulations using this code. The target galaxy is a spherical galaxy with a Hubble profile. The intruder is a selfconsistent disk-halo system. The disk is exponential with a finite thickness, and the halo is nearly spherical and isothermal. This system has a Toomre $Q$ value of about 1.5 and is stable. The mass-ratio of disk to elliptical is $1 / 10$. We have used 5000 particles in the disk, 1000 particles in the halo and 1000 in the elliptical galaxy.

A number of encounters were performed and followed for a few dynamical times after disruption. The present code is not suited for following the evolution long after the disk galaxy is completely disrupted, so we restrict ourselves to the disruption phase. We find that in order to form anything resembling shells, the disruption has to be nearly instantaneous i.e. from a close parabolic or almost radial orbit. If the disruption is slow, the disintegration resembles Roche lobe overflow through the Lagrange point and the particles are scattered widely in phase space. This means that the disk galaxy must be fairly compact so it does not loose its stars too early.

When the disk galaxy has zero thickness, some nearly radial encounters produce sharp edged features. However this happens only in a small number of the encounters that we have simulated. When we perform the same encounters when the disk scale height to scale length is $1 / 10$ as is inferred for our own galaxy, the observed features are much broader and less distinct. James and Wilkinson (1987) find, however, in similar numerical simulations that shells do form even with a finite temperature of the disk. The reason for this discrepancy is not clear. We conclude that Quinn's mechanism will be effective only in a small fraction of galaxy encounters. The shells must have formed by disk stars that have a small velocity dispersion. The sharpness of the shells cannot be explained by phase wrapping of an old, warm disk component. Phase wrapping of a young, cooler component can explain the sharpness of the features.

\section{REFERENCES}

Hernquist, L., Quinn, P., 1986a. Astroph. J., in press.

Hernquist, L., Quinn, P., 1986b. Astroph. J., in press.

Hernquist, L., Quinn, P., 1986c. This volume, p. 467.

James, R. A. and Wilkinson, A., 1987. This volume, p. 471

Malin, D. F., Carter, D., 1980. Nature, 285, 643.

Malin, D. F., Carter, D., 1980. Astroph. J., 274, 534.

Morse, P. M., and Feshbach, H., 1953. Methods of Theoretical Physics, McGrawHill, New York.

Quinn, P., 1984. Astroph. J., 279, 596.

Quinn, P. and Hernquist, L., 1987. This volume, p. 249.

Schweizer, F., 1980 Astroph. J., 252, 303.

Villumsen, J.V., 1982 M.N.R.A.S., 199, 493. 